\title{
Konzeptioneller Rahmen zum Verhältnis formellen und informellen Lernens
}

\section{Matthias Rohs}

Der Artikel setzt sich vor dem Hintergrund der zunehmenden Bedeutung informellen Lernens mit dem Verhältnis formeller, non-formeller und informeller Lernprozesse auseinander. Ausgehend von der Darstellung der Motive für eine stärkere Verbindung unterschiedlicher Lernformen werden zunächst die begrifflichen und definitorischen Zusammenhänge zur Beschreibung des Verhältnisses formellen, non-formellen und informellen Lernens beleuchtet. Darauf aufbauend werden die Schnittstellenprozesse zwischen den Lernformen sowie Rahmenbedingungen für das Zusammenwirken beschrieben. Der Beitrag liefert damit eine Grundlage für die Reflexion pädagogischer Praxis sowie für die Fundierung der empirischen Forschung in diesem Feld.

\section{Motive und Beziehungen}

Die Neuentdeckung informellen Lernens hat die Bildungswissenschaft und die pädagogische Praxis vor die Frage gestellt, wie das damit verbundene Potenzial genutzt werden kann. Sowohl aus bildungspolitischer Perspektive wie auch aus institutioneller Perspektive (von der Schule über die Hochschule bis zur Weiterbildung) werden Konzepte entwickelt, die auf die Herstellung von Bezüge zwischen formellen ${ }^{1}$, non-formellen und informellen Lernprozesse zielen (unten). Dabei können u.a. folgende Motive unterschieden werden:

a) Formelles und informelles Lernen werden als komplementäre Bestandteile der Kompetenzentwicklung betrachtet.

b) Durch eine pädagogische Steuerung (Formalisierung) sollen die Potenziale informellen Lernens nutzbar gemacht werden.

c) Zugangsmöglichkeiten zu (formalen) Abschlüssen sollen erweitert werden.

d) Die Selbststeuerung des Lernprozesses soll betont werden.

Die Vorstellungen des Zusammenwirkens sind dabei eng an das jeweilige Verständnis der Lernformen gebunden. Die begrifflichen Unklarheiten aufgrund der vielfältigen Definitionen informellen Lernens wirken sich auch 
auf die Konzeption und Gestaltung ihres Zusammenwirkens aus. Daher soll im Folgenden der Versuch unternommen werden, dieses Zusammenwirken aus der Perspektive der unterschiedlichen Definitionsansätze differenziert zu beschreiben.

In der Literatur finden sich verschiedene Beispiele zur Gestaltung des Verhältnisses formellen, non-formellen und informellen Lernen, wie z.B.:

- Integration (Dehnbostel, 2002; Svensson, Ellström \& Åberg, 2004; Kepp et al., 2008; Hirning, 2009),

- Verbindung (Elsholz, 2004)

- Bridging (Hofstein \& Rosenfeld, 1996; Eshach, 2007; Cook, Pachler \& Bradley, 2008; Trinder et al., 2008; Feichas,, 2010)

- Connecting (Bull et al., 2008; Dabbagh \& Kitsantas, 2012) oder

- Fusion (Hall 2012)

- Combining (Misko, 2008; Sorfenfrei \& Smolnik, 2013).

Die verschiedenen Blickwinkel könnten a) auf unterschiedliche Verständnisse und Zielsetzungen, b) eine Vielschichtigkeit der Bezüge formellen, non-formellen und informellen Lernens oder c) auf eine mangelnde begriffliche Durchdringung hinweisen. Zur Klärung wird daher im Folgenden zunächst auf grundsätzliche Definitionsansätze und anschliessend auf die Bedingungen des Zusammenwirkens eingegangen. Damit soll zu einer besseren theoretischkonzeptionellen Beschreibung und Gestaltung der Bezüge formellen und informellen Lernens beigetragen werden.

\section{Grundlegende Definitionsansätze}

Angesichts der Vielzahl an Definitonsansätze zur Beschreibung formellen, non-formellen und informellen Lernens soll zunächst eine grundsätzliche Kategorisierung der Ansätze vorgenommen werden. Die Kategorisierung erfolgt dabei in Hinblick auf die Abgrenzung der beiden Lernformen. Diesbezüglich lassen sich drei Definitionsansätze unterscheiden:

\section{Distinktive Ansätze}

Ein erster Ansatz nimmt eine klare Trennung formellen und informelles Lernen anhand einzelner oder mehrerer polarisierender Kriterien ${ }^{2}$ vor. Beispielhaft dafür sind Definitionen, die sich auf den Kontext beziehen: Formelles Lernen findet demnach in der Schule bzw. Einrichtungen des formalen Bildungssystems statt und informelles Lernen ausserhalb davon (z.B. Dewey, 1916 S. 4ff; Dohmen 2001, S. 25; Hager \& Halliday, 2008, S. 1f). Die Unterscheidung verweist damit nicht nur auf eine Differenz, sondern auf auch auf eine gegenseitige Abhängigkeit in der Beschreibung. 


\section{Diskret gestufte Ansätze 3}

Diskret gestufte Ansätze, die in formelles, non-formales und informelles Lernen differenzieren, wurden insbesondere von internationalen Organisationen in die Diskussion eingebracht (Coombs \& Ahmed, 1974, S. 8; Commission oft the European Communities, 2000, S. 8; OECD, 2007, S. 3). Das non-formelle Lernen bildet dabei eine Zwischenkategorie als organisiertes Lernen, welches nicht im formalen Bildungssystem stattfindet. Dabei stellten schon Coombs \& Ahmed (1974) im Hinblick auf die Unterscheidung von formellem und non-formellem Lernen fest: "It should be said, however, that there is no sharp dividing line between them. Moreover, their differences occasionally merge in, hybrid' programs combining significant features of both.» (S. 9).

Neben der generellen Unterscheidung zwischen diesen drei Formen entwickelte La Belle (1982) einen zweidimensionalen Ansatz, der zwischen dem Grad der Formalität in Bezug auf «Educational mode» und «Educational characteristics» unterscheidet (vgl. Abb. 1). Ausschlaggebend dafür war die Feststellung: "In practice, informal, nonformal and formal education should be viewed as predominant modes of learning rather than, as Coombs and Ahmed imply, discrete entities. As modes of emphasis, formal, nonformal, and informal education may exist simultaneously, sometimes in concert with one another and sometimes in conflict." (La Belle 1982, S.162). ${ }^{4}$

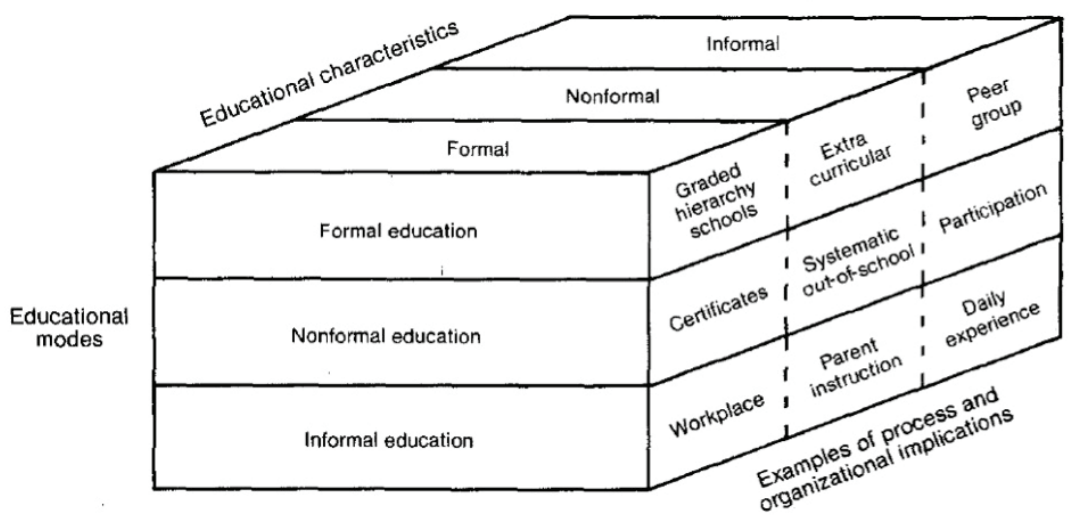

Abbildung 1: The Modes and Characteristics of Education (La Belle, 1982, S. 162)

Kont inuumansätze

Die bei den zuvor vorgestellten Ansätze anklingenden Schwierigkeiten einer klaren Differenzierung zwischen den verschiedenen Lernformen verstärkte sich mit der Berücksichtigung immer weiterer Kriterien (vgl. Colley, Hodkinson \& Malcolm, 2003) und führten zu der Erkenntnis: «that it is not possible to clearly define separate ideal-types of formal and informal learning, which bear any relation to actual learning experiences» (Hodkinson, Colley und Malcolm, 2003, 
S, 14). Vor diesem Hintergrund wurden Definitionen formuliert, die keine klare Trennung der Lernformen vorsehen, sondern von einem Kontinuum zwischen formellem und informellem Lernen ausgehen. Während Stern und Sommerlad (1999) dieses Kontinuum anhand von Beispielen beschreiben, definieren andere Autoren die Ausprägungen anhand einzelner Dimensionen (z.B. Steuerung) für formelles bzw. informelles Lernen (z.B. Knoll, 2002; Rohs, 2008).

\section{Dimensionen des Lernens}

\begin{tabular}{lcr}
$\begin{array}{c}\text { Charakteristika } \\
\text { formelles Lernen }\end{array}$ & $\begin{array}{c}\text { Charakteristika } \\
\text { informelles Lernen }\end{array}$ \\
\hline Lernintention & Intention & Problemlösung \\
\hline organisiertes pad. Angebot & Lernunterstützung & Nachfrage, nicht organisiert \\
\hline fremd gesteuert, festgelegt & Steuerung (Ziele, Inhalte, Zeit) & selbst bestimmt \\
\hline fokussiert & Gegenstand & ganzheitlich \\
\hline bewnsstes Lernen & Bewusstheit & teilweise unbewusstes Lernen \\
\hline Theoriewissen & Lernergebnis & Erfahrungswissen \\
\hline
\end{tabular}

Abbildung 2: Kontinuummodell formellen und informellen Lernens (Rohs 2008, S. 28)

Diese unterschiedlichen definitorischen Sichtweisen sollen folgend mit Bezug zur Beschreibung des Verhältnisses formellen, non-formellen und informellen Lernens beleuchtet werden, d.h. es wird der Frage nachgegangen, wie sich unterschiedliche Definitionsansätze auf die Beschreibung und Gestaltung des Verhältnisses der Lernformen auswirken. Dazu wird zunächst ausführlich auf den Modus der Integration eingegangen, bevor abschliessend die Unterschiede zum Modus der Verbindung erläutert werden.

\section{Definitionsansatz und Beziehungsart}

Integration (lat. integrare) bedeutet so viel wie Vervollständigen oder Wiederherstellen eines Ganzen. In der Erziehungswissenschaft wird der Begriff in organisatorischer, inhaltlich-curricularer und sozialer Dimension thematisiert (Semmerling, 1986, S. 479ff.). Die Verwendung des Begriffs «Integration» im Zusammenhang mit Lernformen adressiert die methodisch-didaktische Ebene und berührt dabei auch soziale Belange einer Integration (z.B. Umgang mit Heterogenität).

In der Literatur existieren zahlreicher Beispiele zur Integration formellen und informellen Lernens (Richardson \& Wolfe, 2001; Otto \& Kutscher, 2004; 
Svensson, Ellström \& Åberg, 2004; Ghislandi, et al. 2013 u.a.), jedoch wird die Frage der Möglichkeiten der Integration formellen und informellen Lernens an sich kaum grundlegend diskutiert. Dabei ist zuerst einmal zu fragen, was worin integriert werden soll. In der Regel ist es Ziel, das informelle Lernen in das formale Bildungssystem bzw. in formal organisierte Bildungsangebote zu integrieren. Dieser Blickwinkel rührt aus der historisch gewachsenen Dominanz formaler Bildung und der als höherwertig betrachteten Lernergebnisse (oftmals in Form von Theoriewissen) her (Bollweg, 2008, S. 49). Auch wenn es in den letzten Jahren zu einer Aufwertung des informellen Lernens und informell erworbener Kompetenzen gekommen ist (Bohlinger, 2009, S. 161), kann aktuell noch nicht von einer Gleichwertigkeit der Lernformen und der damit verbundenen Lernergebnisse gesprochen werden. Eine Integration formellen Lernens in das informelle Lernen erscheint aufgrund der Formlosigkeit, d.h. der schwierigen Fassbarkeit des Informellen schwer denkbar. Dabei kann die «Kolonialisierung der Lebenswelt» (Geissler, 2003) durch das informelle Lernen, wie sie beispielsweise durch die Nutzung von mobilen Lernanwendungen (Apps) propagiert wird (vgl. Gillies, 2013), als solch eine Integration verstanden werden.

Integration setzt dabei voraus, dass die Ausprägungen des formellen bzw. informellen Lernens definitorisch klar zu trennen sind, wie es bei den distinktiven Ansätzen der Fall ist. Dies würde aber bedeuten, dass das informelle Lernen bei einer Integration in einen formalen Kontext formalisiert werden würde und die spezifischen Eigenheiten informellen Lernens verloren gehen würden. Die Bemühungen um eine stärkere «Integration» des informellen Lernens zielen aber u.a. genau auf die Potentiale dieser Lernform, die durch eine Formalisierung in Frage gestellt werden würden: "It became clear during the desk study that the initially proposed idea of integration of informal and formal learning was not in fact desirable as the very process of integration would run the risk of formalising informal practises and may in fact destroy any benefits that informal learning offered by thus doing.» (Trinder et al., 2008, S. 5). 5

In den diskret-gestuften Ansätzen stellt das non-formelle Lernen eine intermediäre Stufe dar, die definitorisch vom formellen und informellen Lernen abgegrenzt wird. Konkret beschreibt das non-formelle Lernen dabei eine grosse Bandbreite an Lernsituationen, die weder eindeutig formell noch informell sind. Dabei können für das non-formelle Lernen sowohl Charakteristika formellen Lernens (z.B. Lernunterstützung) als auch informellen Lernens (z.B. fehlende Zertifizierung) zutreffen. Non-formelles Lernen kann in diesem Sinne auch als Integrationsstufe gesehen werden, die eine Mischung von Kriterien formellen und informellen Lernens darstellt.

Bei den zweidimensional-gestuften Ansätzen (z.B. La Belle, 1982) ist durch die Trennung von Lernkontext und -prozess eine Verschränkung formellen, non-formellen und informellen Lernens konzeptionell angedacht und entsprechend beschreibbar. Damit ist auch eine Integration möglich, ohne die begriffliche Substanz der entsprechenden Lernform zu verändern. Gleichwohl ist davon 
auszugehen, dass der Kontext den Lernprozess beeinflusst, d.h. das nicht-formale Lernen in formalen Kontexten anders verläuft als in informellen Kontexten.

Unter dem Blickwinkel eines Kontinnuumansatzes ist die Forderung einer Integration nicht plausibel, da diese Definitionsansätze bereits davon ausgehen, dass sich das Verhältnis formellen und informellen Lernen als ein untrennbares, integriertes darstellt: "It is important not to see informal and formal attributes as somehow separate, waiting to be integrated. This is the dominant view in the literature, and it is mistaken. Thus, the challenge is not to, somehow, combine informal and formal learning, for informal and formal attributes are present and inter-related, whether we will it so or not.» (Hodkinson, Colley \& Malcom, 2003, S. 314). Integration würde in diesem Sinne eine Betonung der Verbindung von Charakteristika der einen Lernform mit denen der anderen Lernform bedeuten, wie z.B. offenere Lernziele, stärkere Selbststeuerung in formellen Lernsettings oder eine stärkere Steuerung und Strukturierung informellen Lernens (z.B. durch pädagogische Beratung oder Bereitstellung von Lernressourcen). Beispielhaft für eine Integration im Bereich des betrieblichen Lernens wären arbeitsintegrierte Lernformen (z.B. Arbeits- und Lernaufgaben) und lernförderliche Arbeitsformen (z.B. Job Rotation) zu nennen (Dehnbostel, 2007), die sowohl Eigenschaften formalen als auch informellen Lernens besitzen.

Werden diese drei grundsätzlichen Definitionsansätze nun zusammenfassend betrachtet, so zeigt sich, dass beim erste Ansatz die Integration beider Lernformen nicht möglich ist, sondern sich die Verbindung über eine Output-Input-Beziehung realisieren lässt, d.h. Ergebnisse informellen Lernens können Grundlage formeller Lernprozesse sein und umgekehrt. Bei den gestuften Ansätzen ist die Integration formellen und informellen Lernens eine Zwischenstufe des non-formellen Lernens (kategoriale Integration) oder eine Kombination unterschiedlicher Dimensionen (dimensionale Integration). Bei den Kontinuumansätzen wird hingegen davon ausgegangen, dass eine Integration stets gegeben ist, wobei sich der Grad der Integration unterscheidet (kontinuierliche Integration).

Neben dem Anspruch der Integration werden in der Literatur, wie einführend erwähnt, noch Begriffe wie "connecting», «bridging» oder "combining» verwendet, die das Zusammenwirken formellen und informellen Lernens beschreiben sollen. Diese drücken semantisch eher eine Verbindung aus, da sie kein hierarchisches Verhältnis beschreiben oder eine Richtung des Prozesses vorgeben. Beide Lernformen werden in diesem Zusammenhang als gleichwertig betrachtet. Zudem ist es nicht Ziel, etwas anzugleichen oder zu verändern, sondern die Elemente in ihrer Charakteristik zu erhalten. Bei einer Verwendung dieser Begriffe muss daher davon ausgegangen werden, dass formelles, non-formelles und informelles Lernen klar zu unterscheiden sind und das Ziel vor allem in der Gestaltung der Schnittstellen zwischen diesen Lernformen liegt. Dieses Verständnis setzt eine klare definitorische Trennung der Lernformen voraus, wie sie bei distinktiven und gestuften Ansätzen gegeben ist. Die Verbindung könnte daher als Folge von «Lernepisoden» (Tough, 1950) beschrieben werden, 
die formellen oder non-formellen bzw. informellen Charakter besitzen. Im Gegensatz zu den integrativen Ansätzen, bei denen die formellen Anteile steuernd wirken, ist die Verbindung formellen und informellen Lernens dadurch geprägt, dass informelles Lernen stattfindet und inhaltliche Anschlussstellen für formelles Lernen bietet. Bei den zweidimensional-gestuften Ansätzen kann die Verbindung als Kombination kontextuell und prozessual unterschiedlicher Lernepisoden beschrieben werden. Im Verständnis eines Kontinuums formellen und informellen Lernens ist die Forderung einer Verbindung nicht plausibel, da es keine Trennung der Lernformen gibt. Es wäre lediglich eine Verbindung von Lernformen mit unterschiedlich starker formeller oder informeller Ausprägung möglich.

Zusammenfassend kann damit festgestellt werden, dass der zugrundeliegende Definitionsansatz Auswirkungen auf die Beschreibung des Verhältnisses formellen, non-formellen und informellen Lernens hat und dass die Vorstellung des Zusammenwirkens mit spezifischen Definitionsansätzen in Verbindung steht (vgl. Tabelle 1).

\section{Tabelle 1: Beziehungsform und Definitionsansatz formellen, non-formellen und} informellen Lernens

\begin{tabular}{|l|c|c|c|}
\hline Beziehungsform & Definitionsansatz & Kontinuumansätze \\
\hline & Distinktive Ansätze & Gestufte Ansätze & $\begin{array}{c}\text { kontinuierliche } \\
\text { Integration }\end{array}$ \\
\hline Integration & nicht möglich & $\begin{array}{c}\text { kategoriale oder dimen- } \\
\text { sionale Integration }\end{array}$ & $\begin{array}{c}\text { nicht plausibel } \\
\text { Verbindung }\end{array}$ \\
\hline
\end{tabular}

Neben der definitorischen Betrachtung, die grundlegend für die Beschreibung des Verhältnisses der Lernformen ist, stellt sich aus pädagogischem Blickwinkel die Frage der Gestaltbarkeit des Zusammenwirkens formellen, non-formellen und informellen Lernens unter dem Modus der Integration und Verbindung, worauf im Folgenden näher eingegangen werden soll.

\section{Prozesse und Bedingungen}

Um das Zusammenwirken formellen, non-formellen und informellen Lernens zu beschreiben, ist zum einen auf die Prozesse einzugehen, die an den Schnittstellen der Lernformen wirksam werden und zum anderen auf die Rahmenbedingungen, die das Zusammenwirken beeinflussen. An den Schnittstellen werden grundsätzlich zwei Prozesse unterschieden: 
a) Als ein Prozess wird von verschiedenen Autoren die Reflexion hervorgehoben (Overwien, 2003; Svensson \& Ellström, 2004; Kirchhof, 2007). Reflexion kann dabei als Prozess der Überführung impliziten Wissens in explizite subjektive Theorien (Neuweg, 2002, S. 26) verstanden werden. Das sich daraus ergebende reflexive Erfahrungswissen ist dann produktiv nutzbar und in einem Verhältnis zu formell erworbenem wissenschaftlichem Theoriewissen zu diskutieren. Die Reflexion beschreibt damit einen Prozess der Verbindung informellen und formellen Lernens, der durch Problem- und Krisensituationen oder auch durch bewusste pädagogischer Intervention angestossen werden kann. ${ }^{6}$

b) Aus dem Blickwinkel formellen Lernens kann der verbindende Prozess als Steuerung der Wahrnehmung durch Schemata beschrieben werden. Die Aneignung und das Verständnis von Schemata werden breit diskutiert. Für die Verbindung formellen und informellen Lernens sind vor allem die Wissensschemata (vgl. Kopp \& Mandl, 2005) anschlussfähig. Sie werden durch Experten an die Lernenden vermittelt (formelles Lernen) und umfassen neben konkrete Objekte und Gegenstände auch abstrakte Zusammenhänge wie z.B. Theorien (vgl. Abb. 3).

Das pädagogische Anliegen, ein Zusammenwirken beider Lernformen zu unterstützen, wird durch verschiedene Rahmenbedingungen beeinflusst. Als zentrale Faktoren sind dabei die Zeit, der Inhalt und der Kontext zu betrachten:

\section{a) zeitliche Aspekte des Zusammenwirkens}

Aus zeitlicher Perspektive stellt sich die Frage, in welcher Abfolge die Lernformen aufeinander aufbauen. Aus einer distinktiven Betrachtungsweise heraus war für Dewey das informelle Lernen das natürliche und grundlegende Lernen; es soll nicht nur als Vorbild, sondern auch als Ressource für das schulische Lernen dienen (Gonon, 2002, S. 18), d.h. die formellen Lernprozesse sollen auf den informellen Lernprozessen aufbauen. In ähnlicher Weise wird in der Erwachsenenbildung der Rückgriff auf die Erfahrungen der Teilnehmenden als wesentlichen Voraussetzung für eine erfolgreiche Gestaltung von Lernprozessen betrachtet. Diese Sichtweise drückt sich u.a. im Ansatz der Biographieorientierung aus, nach der institutionalisierte Bildungsangebote bei den Teilnehmenden auf eine Erfahrungs- und Lerngeschichte treffen (Dausien \& Alheit, 2005, S. 28) die es zu berücksichtigen gilt.

Das formelle Lernen hingegen ist mit einem Transferproblem konfrontiert, d.h., dass das erworbene Wissen durch die Lernenden nicht in der Praxis angewendet werden kann. So kamen auch Staudt und Kley (2001) bei der Betrachtung informellen Lernens in betrieblichen Kontexten zu dem Schluss, dass «die Wissensvermittlung in formellen Lernarrangements stets nur die Anfangsgründe eines Lernprozesses bereitstellen»(S. 41). Dieser muss dann in einem Transferprozess in der Praxis fortgesetzt werden, oder wie Svensson und Ellström (2009) es ausdrücken: «Formal Education needs to backed up by 
informal learning in order to be effective.» (S. 481). Diese Sichtweise ist eng mit kompetenzorientierten Lernzielen verbunden.

Bei der Betrachtung der zeitlichen Abfolge zeigt sich demnach, dass unter distinktiven Ansätzen das Zusammenwirken formellen, non-formellen und informellen Lernens in keine Richtung determiniert ist, sondern als kontinuierlicher Prozess des wechselseitigen Aufeinanderfolgens verstanden werden kann, der spiralförmig zu einer Steigerung des Wissen- und Kompetenzniveaus führt.

\section{b) Inhaltliche Aspekte des Zusammenwirkens}

Dehnbostel (2007) geht in seinem Modell der betrieblichen Wissensarten davon aus, dass informelles Lernen vor allem zu Erfahrungswissen führt, während über formelles Lernen im Wesentlichen Theoriewissen angeeignet wird. Für die Aneignung beruflicher Handlungskompetenz schliesst er daraus auf die Notwendigkeit der Verbindung beider Lernformen (S. 41f).

Die Verbindung setzt dabei einen inhaltlichen Bezug formeller und informeller Lernepisoden voraus. Diese Kopplung wird z.B. im Rahmen dualer Ausbildungs- und Studienkonzepte angestrebt. In formellen Kontexten erworbene theoretische Inhalte sollen durch informelle Lernerfahrungen in der praktischen Umsetzung ergänzt bzw. Praxiserfahrungen nachfolgend durch wissenschaftliches Wissen in formellen Kontexten fundiert werden. Hierdurch ergibt sich eine inhaltliche Verbindung von Theorie- und Praxiswissen, die zu entsprechender Handlungskompetenz führt.

Die Gestaltung dieser inhaltlichen Kopplung ist didaktisch anspruchsvoll, da sich informelle Lernprozesse weitgehend einer Steuerung entziehen. Die Herstellung der inhaltlichen Verknüpfung liegt daher in der Hand der Lernenden. Die Rolle der pädagogischen Begleitung liegt vor allem in der Gestaltung des informellen Lernumfeldes, der Bezugnahme zu individuellen Lernerfahrungen und in der Befähigung der Lernenden zur Selbststeuerung ihres Lernprozesses.

Der Unterschied in der inhaltlichen Verknüpfung zwischen den verschiedenen Ansätzen ist darin zu sehen, dass bei einer distinktiven Interpretation die zeitlichen Phasen formellen und informellen Lernens tendenziell länger sind als in den anderen Ansätzen, da für eine inhaltliche Verbindung nicht zwangsläufig ein (aufwändiger) Kontextwechsel notwendig ist.

\section{c) Kontextuelle Aspekte des Zusammenwirkens}

Formelle, non-formelle und informelle Lernprozesse werden in der Regel mit bestimmten Kontexten verbunden. So überwiegt das formelle Lernen in Einrichtungen des formalen Bildungssystems, das non-formelle Lernen in Einrichtungen ausserhalb des formalen Bildungssystems und das informelle Lernen im Rahmen nicht-organisierten Lernens. Nach diesem distinktivem Verständnis sind Lernformen bestimmten Kontexten zugeordnet. Dementsprechend erfordert die Verknüpfung der Lernformen eine Verbindung der Lernkontexte. Diese Verbindung kann über das Lernen in verschiedenen Organisationen oder organi- 
sationalen Subsystemen hergestellt werden. Eine solche Verbindung besteht beispielsweise zwischen dem Lernen im Arbeitsprozess (informelles Lernen) und und innerbetrieblichen Angeboten der Weiterbildung in Seminarform (non-formelles Lernen) oder zwischen Schule (formelles Lernen) und Sportverein (non-formelles Lernen). Derartige Kopplungen zwischen Organisationen oder organisationalen Subsystemen können in Hinblick auf Dauer und Frequenz unterschiedlich intensiv gestaltet sein.

Ist ein integratives Verständnis des Zusammenwirkens der Ausgangspunkt, so ergibt sich für Organisationen, in denen formell gelernt wird, die Notwendigkeit, informelles Lernen zu ermöglichen. Die Förderung kann sich dabei auf inhaltliche, methodische, örtliche u.a. Dimensionen beziehen. Dabei ist zu unterscheiden, ob es eine allgemeine Förderung des informellen Lernens ist (z.B. über das Einräumen von Lernzeiten), oder ob das Lernen auf ein bestimmtes Ziel hin gefördert wird (z.B. über die Bereitstellung von speziellen Lernressourcen).

Bei der Förderung des informellen Lernens in Unternehmen werden dabei zum einen die Arbeit selbst und zum anderen die Arbeitsbedingungen lernförderlich gestaltet. Dabei sind z.B. Kriterien wie die Vollständigkeit der Arbeitshandlung, Abwechslungsreichtum, Anforderungsniveau und -vielfalt von Bedeutung. Darüber hinaus soll die Arbeit auch Autonomieerleben und soziale Unterstützung ermöglichen (vgl., Dehbostel, 2007, Frieling et al., 2006). Neben diesen konkreten Gestaltungshinweisen gilt es allgemein, die Lernkultur zu verändern, d.h. den Teil der Organisationskultur, der den Stellenwert des Lernens im Unternehmen widerspiegelt (vgl. Sonntag, Stegmaier, Schaper \& Friebe, 2004). Die Lernkultur umfasst dabei auf normativer Ebene lernbezogene Werte, Normen und Einstellungen sowie auf strategischer Ebene in den Rahmenbedingungen, die der längerfristigen Lernunterstützung dienen (ebd., S. 107). Aber auch digitale Medien können bei der kontextuelle Verbindung oder Integration eine besondere Rolle spielen, da sie ortsunabhängiges Lernen unterstützen. Insbesondere mobile learning kann dazu beitragen räumliche Grenzen zu durchbrechen und Lernen ausserhalb und innerhalb formeller Bildungskontexte zu verbinden (Rohs, 2013).

Zusammenfassend werden in Abb. 3 die prozessualen und kontextuellen Bezüge formellen, non-formellen und informellen Lernens dargestellt. Danach sind es vor allem die Prozesse der Reflexion und Wahrnehmungssteuerung, die als Schnittstellenprozesse zwischen den Lernformen wirken. Als Rahmenbedingungen wirken zeitliche, inhaltliche sowie kontextuelle Faktoren. Das non-formelle Lernen nimmt eine Zwischenstellung ein, das als integrierende Form mit Charakteristika formellen und informellen Lernens beschrieben werden kann. Dabei wird davon ausgegangen, dass formelles Lernen dominant aber nicht ausschliesslich zur Entwicklung von Theoriewissen führt, während informelles Lernen vor allem zur Generierung von Erfahrungswissen führt. 


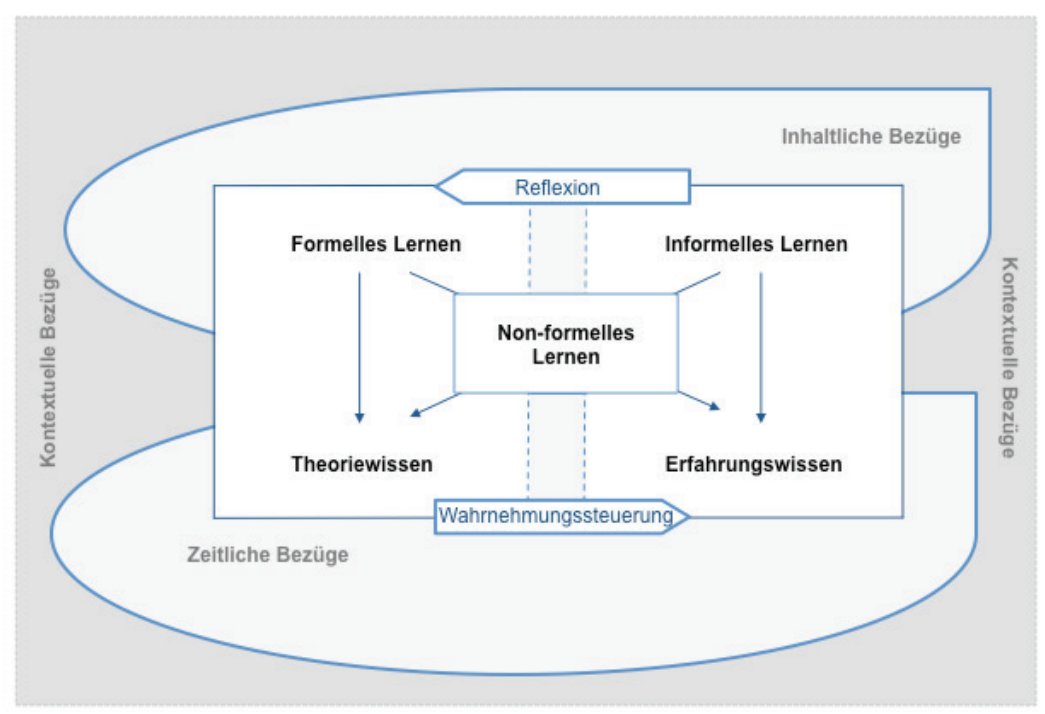

Abbildung 3: Bezüge formellen, non-formellen und informellen Lernens

\section{Faz it}

Der Artikel hat sich vor dem Hintergrund einer zunehmenden Bedeutung informellen Lernens mit dem Verhältnis formellen, non-formellen und informellen Lernens auseinandergesetzt und eine systematischen Betrachtung und Zusammenführung der begrifflichen und definitorischen Grundlagen sowie der Mechanismen und Rahmenbedingungen des Zusammenwirkens dieser Lernformen vorgenommen. Auf eine weitere Differenzierungen informellen Lernens und auf die Beschreibung von Bezügen zur Sozialisation wurden dabei verzichtet, da sie die Komplexität der Betrachtungen zusätzlich erhöht hätten. Im Ergebnis liefert der Beitrag einen konzeptionellen Rahmen zu Beschreibung und Gestaltung der Beziehungen zwischen diesen Lernformen.

Die Verbindung oder Integration formellen und informellen Lernens ist kein Selbstzweck. Trotz der einführend erwähnten Motive, die grundsätzlich für eine pädagogische Gestaltung der Beziehung zwischen den Lernformen sprechen, bedarf es in jedem Einzelfall einer Abwägung der Relevanz informellen als auch formellen Lernens für die jeweilige Zielsetzung sowie möglicher Nachteile, die eine stark ode schwach ausgebildete Formalisierung des Lernprozesses mit sich bringt.

Die Herausforderung besteht abschliessend darin, die erkannten Mechanismen und Einflussfaktoren in eine konkrete pädagogische Praxis umzusetzen. Damit ist nicht nur die mikrodidaktische Ebene angesprochen, sondern 
grundsätzlich auch die Frage des Stellenwerts informellen Lernens in institutionellen Bezügen oder im Bezug zum Bildungssystem, wie sie im Kontext der Anerkennung informell erworbener Kompetenzen diskutiert wird. Das Verhältnis formellen, non-formellen und informellen Lernens ist somit auf allen pädagogischen Handlungsebenen anzusprechen und zu reflektieren.

\section{Anmerkungen}

1 «Formell und formal sowie non-formal bzw. non-formell» werden synonym verwandt. Richtig müsste es eigentlich «formal» und «informal» heissen (die Form betreffend).

2 Colley, Hodkinson \& Malcolm (2003) identifizieren mehr als 20 Kriterien und kategorisierten diese in Process, Location Setting, Purposes und Content. Straka (2004) unterscheidet ebenfalls auf der Basis dieser Kriterien «Macro-Level», «Micro-Level», «External Conditions» und "Activities oft the learner».

3 Unberücksichtigt bleiben hier Betrachtungen zur Binnendifferenzierung informellen Lernens, wie sie Marsick \& Watkins (1990), Schugurensky (2000) oder Eraut (2004) entwickelt haben.

4 In vergleichbarer Weise geht Straka (2004, S. 12) davon aus, dass explizites und implizites Lernen unter formellen, non-formellen und informellen Bedingungen stattfindet.

5 Studien zu Online-Communities führen ebenfalls diese Bedenken auf, gehen aber davon aus, dass eine Formalisierung von Online-Communities möglich und für deren nachhaltige Existenz von Bedeutung ist (Jahnke 2009, S. 5f). Dabei wird deutlich, dass die Formalisierung nicht als die Umwandlung einer Lernform in eine andere Lernform gedacht ist, sondern als Ausbalancierung formeller und informeller Anteile des Lernens.

6 Reber (1993) zeigte im Zusammenhang mit seinen Forschungsarbeiten, dass auch unbewusstes, implizites Wissen spätere Handlungen beeinflusst.

\section{Literatur}

Bohlinger, S. (2009). Bildungspolitische Implikationen informellen Lernens. In bildungsforschung, Jg. 1, 159-186.

Bull, G., Thompson, A., Searson, M., Garofalo, J., Park, J., Young, C., \& Lee, J. (2008). Connecting Informal and Formal Learning Experiences in the Age of Participatory Media. In Contemporary Issues in Technology and Teacher Education, 8(2). pp. 100-107

Colley, H., Hodkinson, P., \& Malcom, J. (2003). Informality and formality in learning: A report for the Learning and Skill Reserach Centre. London: Learning and Skills Research Center.

Commission oft the European Communities (2000). A Memorandum of Lifelong Learning. Brussels.

Coombs, P. H., \& Ahmed, M. (1974). Attacking Rural Povertry: How nonformal education can help. Baltimore and London: World Bank.

Cook, J., Pachler, N., \& Bradley, C. (2008). Bridging the Gap? Mobile Phones at the Interface between Informal and Formal Learning. In Journal of the Research Center for Educational Technology, 4(1), pp. 3-18.

Dabbagh, N., und Kitsantas, A. (2012). Personal Learning Environments, social media, and self-regulated learning: A natural formula for connecting formal and informal learning. In The Internet and Higher Education, 15(1), pp. 3-8.

Dehnbostel, P. (2002). Modelle arbeitsbezogenen Lernens und Ansätze zur Integration formellen und informellen Lernens. In M. Rohs (Hrsg.), Arbeitsprozessintegriertes Lernen. Neue Ansätze für die berufliche Bildung (S. 37-57). Münster: Waxmann Verlag.

Dehnbostel. P. (2007). Lernen im Prozess der Arbeit. Münster: Waxmann.

Dewey, J. (1916): Democracy and Education. New York: The Macmillan Company. 
Dohmen, G. (2001). Das informelle Lernen: Die internationale Erschliessung einer bisher vernachlässigten Grundform menschlichen Lernens für das lebenslange Lernen aller. BMBF: Bonn.

Elsholz, U. (2004). Lernnetzwerke zur Verbindung formellen und informellen Lernens, In: P. Dehnbostel \& P. Gonon (Hrsg.), Informell erworbene Kompetenzen - Grundlegungen und Forschungsansätze (S. 97-109). Bielefeld: W. Bertelsmann.

Eraut, M. (2004). Informal learning in the workplace. Studies in Continuing Education, 26(2), 247-273.

Eshach, H. (2007). Bridging In-school and Out-of-school Learning: Formal, Non-Formal, and Informal Education. In Journal of Science Education and Technology, 16(2), pp. 171-190.

Feichas, H. (2010). Bridging the gap: Informal learning practices as a pedagogy of integration. In British Journal of Music, 27(1), pp. 47-58.

Frieling, E., Bernhard, H., Bigalk, D. \& Müller, R.F. (2006). Lernen durch Arbeit. Entwicklung eines Verfahrens zur Bestimmung der Lernmöglichkeiten am Arbeitsplatz. Münster: Waxmann.

Geissler, Kh. A. (2003). Alle lernen alles - die Kolonialisierung der Lebenswelt durch das informelle Lernen. In W. Wittwer \& S. Kirchhof (Hrsg.), Informelles Lernen und Weiterbildung: Neue Wege der Kompetenzentwicklung (S. 127 - 141). Neuwied: Luchterhand.

Ghislandi, P., Ierardi, M. G., Leo, T., \& Spalazzi, L. (2013). Guest Editorial: Innovative Technologies for the Seamless Integration of Formal and Informal Learning. Educational Technology \& Society, 16 (1), 1-3.

Gillies, C. (2013). Die App Ära kommt, ManagerSeminare, Heft 186, S. 52-56.

Gonon, P. (2002). Informelles Lernen - ein kurzer historischer Abriss von John Dewey zur heutigen Weiterbildung. In P. Dehnbostel \& P. Gonon (Hrsg.), Informelles Lernen eine Herausforderung für die berufliche Aus- und Weiterbildung (S. 13-20). Bielefeld: W. Bertelsmann.

Hall, R. (2012). Towards a Fusion of Formal and Informal Learning Environments: the Impact of the Read/Write Web. In M. Ciussi \& G. Freitas (Hrsg.), Leading Issues in e-Learning Research (S. 19-46). Reading: Academic Publishing International Limited.

Hager, P., \& Halliday, J. (2009). Rediscovering Informal Learning: Wisdom, Judgement and Community. Dordrecht: Springer.

Hirning, A. (2009). Integration formellen und informellen Lernens. In A. Hohenstein \& K. Wilbers (Hrsg.), Handbuch E-Learning. Wolters-Kluwer.

Hodkinson, P., Colley, H. \& Malcolm, J. (2003). The interrelationships between informal and formal learning, In Journal of Workplace Learning, 15 (7/8), pp. 313-318.

Hofstein, A., \& Rosenfeld, S. (1996). Bridging the Gap Between Formal and Informal Science Learning. In Studies in Science Education, 28(1). pp. 87-112

Jahnke, I. (2009): The Process of Digital Formalization in Sociotechnical Learning Communities - Needed or Overloaded? In: C. O’Malley, D. Suthers P. Reimann \& A. Dimitracopoulou, (Hrsg.), Computer Supported Collaborative Learning Practices. CSCL 2009 Conference Proceedings. S. 287-291.

Kirchhof, S. (2007): Informelles Lernen und Kompetenzentwicklung für und in beruflichen Werdegängen: dargestellt am Beispiel einer qualitativ-explorativen Studie zu in-formellen Lernprozessen Pflegender und ihrer pädagogisch-didaktischen Implikationen für die Aus-und Weiterbildung. Münster. Waxmann.

Knoll, J. (2002). Erneut dräut Lämpels Zeigefinger: Zur Rettung des informellen und des Alltagslernens von manchen ihrer Verfechter, In QUEM-Bulletin, 2, S. 16

Kopp, B., \& Mandl, H. (2005). Wissensschemata Forschungsberichte. München: Institut für Pädagogische Psychologie, LMU München.

Kepp, S.-J., Schorr, H., Womser-Hacker, C., \& Lenz, F. (2008). Chatten kann jede/r ;-). Integration von informellen Lern- und Kommunikationswegen und Social Software in 
ein Blended-Learning-Konzept für Lehramtsstudierende im Bereich Englische Kulturwissenschaften. In S. Zaucher, P. Baumgartner, E. Blaschitz \& A. Weissenbäck (Hrsg.), Offener Bildungsraum Hochschule: Freiheiten und Notwendigkeiten (Band 48). Münster: Waxmann.

Marsick, V. J., \& Watkins, K. E. (1990). Informal and incidental learning in the workplace. New York, NY: Routledge.

Misko, J. (2008). Combining formal, non-formal and informal learning for workforce skill development. NCVER - Australian Industrie Group.

OECD (2007). Terms, Concepts and Models for Analysing the Value of Recognition Programmes. Vienna.

Otto, H.-U. \& Kutscher, N. (Hrsg.) (2004). Informelle Bildung Online: Perspektiven für Bildung, Jugendarbeit und Medienarbeit. Weinheim \& München: Juventa Verlag.

Reber, A. S. (1993). Implicit learning and tacit knowledge: an essay on the cognitive unconscious. Oxford: Oxford University Press.

Richardson, L. D. \& Wolfe, M. (Hrsg.) (2001). Principles and Practice of Informal Education: Learning through life. London \& New York: RoutledgeFalmer.

Rohs, M. (2008). Connected Learning: Zur Verbindung formellen und informellen Lernens in der IT-Weiterbildung. Saarbrücken: VDM-Verlag.

Rohs, M. (2013). Informelles Mobiles Lernen, In: de Witt, C. \& Sieber, A. (Hrsg.), Mobile Learning: Potenziale, Einsatzszenarien und Perspektiven des Lernens mit mobilen Endgeräten (S. 75-100). Wiesbaden. Springer VS

Schugurensky, D. (2000). The Forms of Informal Learning: Towards a Conceptualization of the Field. WALL Working Paper (Vol. 19): SSHRC Research Network.

Semmerling, R. (1986). Integration, In Haller, H.-D. \& Meyer, H. (Hrsg.), Handbuch und Lexikon Erziehung. Band 3: Ziele und Inhalte der Erziehung und des Unterrichts (S. 479-488). Stuttgart: Klett-Cotta.

Sonntag, Kh., Stegmaier, R., Schaper, R. \& Friebe, J. (2004): Dem Lernen im Unternehmen auf der Spur: Operationalisierung von Lernkultur. Unterrichtswissenschaft, 32 (2), S. 104-127

Sorfenfrei, C. \& Smolnik, S. (2013). Towards combining formal and informal learning paths to enhance competence development success. Konferenzbeiträge der 7. Konferenz Professionelles Wissensmanagement. - 15. März 2013, Passau. BerlIn Gito-Verl. S. 24-39

Staudt, E., \& Kley, T. (2001). Formelles Lernen - informelles Lernen - Erfahrungslernen. Wo liegt der Schlüssel zur Kompetenzentwicklung von Fach- und Führungskräften? Eine kompetenzbiografische Studie beruflicher Innovationsprozesse, In Berichte der angewandten Innovationsforschung (Band 193).

Stern, E., \& Sommerlad, E. (1999). Workplace learning. Culture and performance. London: Institute of Personnel and Development.

Straka, G. A. (2004). Informal Learning: Genealogy, Concepts, Antagonism and Questions ITB-Forschungsberichte (Vol. 15).

Svensson, L., Ellström, P.-E., \& Åberg, C. (2004). Integrating formal and informal learning at work. In Journal of Workplace Learning, 16(8), pp. 479-491.

Tough, M. S. (1950). Informal Adult Education: A Guide for Administrators, Leaders, and Teachers. New York: Association Press.

Trinder, K., Guiller, J., Margaryan, A., Littlejohn A. \& Nicol, D. (2008). Learning from digital natives: bridging formal and informal learning. In The Higher Education Academy. o.S.

Schlagworte: Informelles Lernen, formelles Lernen, Integration, Verbindung, Theorie 


\section{Un cadre conceptuel pour penser la relation entre apprentissage formel et informel}

\section{Résumé}

Dans un contexte où l'apprentissage formel a de plus en plus d'importance, cet article traite de la relation entre les processus d'apprentissage formel, non formel et informel. Sur la base d'un exposé des différents motifs en faveur d'une interdépendance plus forte entre apprentissage formel et informel, les fondements conceptuels et définitionnels de la relation entre l'apprentissage formel, non formel et informel sont présentés et clarifiés. L'article décrit ensuite les processus d'interface entre les divers modes d'apprentissage et les conditions qui rendent leurs interactions constructives. Il fournit ainsi une base pour la réflexion sur les pratiques pédagogiques actuelles et pour le fondement de recherches empiriques dans ce domaine.

Mots-clés: Informel, formel, apprentissage, intégration, connexion, théorie

\section{Un quadro concettuale per la relazione tra apprendimento formale e informale}

\section{Riassunto}

Il presente articolo si occupa del rapporto tra processi di apprendimento formale e informale sullo sfondo dellimportanza crescente dell sapprendimento informale. A partire dallesposizione dei diversi motivi a favore di una più forte integrazione di apprendimento formale e informale vengono messi in luce i presupposti concettuali che possono essere impiegati per la descrizione del rapporto di tali forme di apprendimento. Il saggio offre con ciò una base sia per la riflessione sulla prassi pedagogica attuale sia per la fondazione della ricerca empirica in tale ambito.

Parole chiave: Informale, formale, apprendimento, integrazione, rapporto, teoria 


\section{A conceptual framework for the relationship between formal and informal learning}

\section{Summary}

Increasing importance is presently being devoted to informal learning. In this context, this article discusses the relationship among formal, non-formal and informal learning. Based on different arguments for a stronger interdependence between formal and informal learning, the conceptual and definitional backgrounds of the relationships of formal, non-formal and informal learning are described and clarified. Following, the paper presents mediating processes between these learning modes, as well as the conditions that foster their interactions and make them constructive. The paper thus provides a basis to think educational practices and to found empirical research in this field.

Keywords: Informal, formal, learning, integration, connection, theory 